\title{
THE DRAZIN INVERSE THROUGH THE MATRIX PENCIL APPROACH AND ITS APPLICATION TO THE STUDY OF GENERALIZED LINEAR SYSTEMS WITH RECTANGULAR OR SQUARE COEFFICIENT MATRICES*
}

\author{
GRIGORIS I. KALOGEROPOULOS ${ }^{\dagger}$, ATHANASIOS D. KARAGEORGOS ${ }^{\dagger}$, AND \\ ATHANASIOS A. PANTELOUS ${ }^{\dagger}$
}

\begin{abstract}
In several applications, e.g., in control and systems modeling theory, Drazin inverses and matrix pencil methods for the study of generalized (descriptor) linear systems are used extensively. In this paper, a relation between the Drazin inverse and the Kronecker canonical form of rectangular pencils is derived and fully investigated. Moreover, the relation between the Drazin inverse and the Weierstrass canonical form is revisited by providing a more algorithmic approach. Finally, the Weierstrass canonical form for a pencil through the core-nilpotent decomposition method is defined.
\end{abstract}

Key words. Drazin Inverse, Matrix pencil theory, Generalized linear systems.

AMS subject classifications. 15A09, 15A22.

1. Introduction. In this paper, our main purpose is to study the relation between the Drazin inverse (both for rectangular and square constant coefficient matrices) and the relevant matrix pencil approach for the solution of autonomous singular (or regular) linear differential systems of the form

$$
F \underline{x}^{\prime}(t)=G \underline{x}(t)
$$

or, in the discrete analogue, autonomous singular (or regular) linear difference systems of the form

$$
F \underline{x}_{k+1}=G \underline{x}_{k},
$$

where $\underline{x} \in \mathbb{C}^{n}$ is the state control vector and $F, G \in \mathbb{C}^{m \times n}$ (or $F, G \in \mathbb{C}^{n \times n}$ ). For details, see e.g., $[2,3]$ and also [5, 8]. In many applications (e.g., circuit systems [7], the multi-input multi-output (MIMO) Leontief model in Economy [2], the Leslie population model [2]) it is necessary to solve (1.1) and (1.2) considering the two special cases when the matrices are rectangular. If $m>n$ (i.e., more equations

${ }^{*}$ Received by the editors December 21, 2007. Accepted for publication February 25, 2008. Handling Editor: Michael J. Tsatsomeros.

${ }^{\dagger}$ Department of Mathematics, University of Athens, Panepistimiopolis, 15784 Athens, Greece (gkaloger@math.uoa.gr, athkar@math.uoa.gr, apantelous@math.uoa.gr). 
than unknowns parameters) the systems (1.1) and (1.2) are called over-determined; otherwise, if $m<n$ (more unknowns parameters than equations), they are called under-determined.

Given the constant matrices $F, G \in \mathbb{C}^{m \times n}$ and an indeterminate $s$, the pencil $s F-G$ is called regular when $m=n$ and $\operatorname{det}(s F-G) \neq 0$. In any other case, the pencil is called singular, i.e., when $m \neq n$ or $m=n$ with $\operatorname{det}(s F-G) \equiv 0$.

Definition 1.1. [8] The pencil $s F-G$ is said to be strictly equivalent to the pencil $s F_{1}-G_{1}$ if and only if $P(s F-G) Q=s F_{1}-G_{1}$, where $P \in \mathbb{C}^{m \times n}, Q \in \mathbb{C}^{n \times n}$, and $\operatorname{det} P$, $\operatorname{det} Q \neq 0$.

A class of strict equivalence, say $\mathcal{E}_{s}(s F-G)$, is characterized by a uniquely defined element, known as the complex Kronecker canonical form, $s F_{k}-Q_{k}$ (see [4]), which is specified by the complete set of invariants of $\mathcal{E}_{s}(s F-G)$.

Unlike the case of regular pencils, the characterization of singular pencils requires the definition of additional sets of invariants known as the minimal indices. Let us assume that $m \neq n$ and $r=\operatorname{rank}_{\mathbb{R}(s)}(s F-G) \leq \min \{m, n\}$, where $\mathbb{R}(s)$ denotes the field of rational functions having real coefficients. Then, the equations

$$
(s F-G) \underline{x}(s)=\underline{0} \text { and } \underline{\psi}^{t}(s)(s F-G)=\underline{0}^{t}
$$

have solutions $\underline{x}(s)$ and $\underline{\psi}(s)$ which are vectors in the rational vector spaces

$$
\mathcal{N}_{r}(s) \triangleq \mathcal{N}_{r}(s F-G) \text { and } \mathcal{N}_{l}(s) \triangleq \mathcal{N}_{l}(s F-G)
$$

respectively, where

$$
\begin{gathered}
\mathcal{N}_{r}(s) \triangleq\left\{\underline{x}(s) \in \mathbb{C}^{n}(s):(s F-G) \underline{x}(s)=\underline{0}\right\}, \\
\mathcal{N}_{l}(s)=\left\{\underline{\psi}(s) \in \mathbb{C}^{n}(s): \underline{\psi}^{t}(s)(s F-G)=\underline{0}^{t}\right\} .
\end{gathered}
$$

Obviously, $\mathcal{N}_{r}(s)$ and $\mathcal{N}_{l}(s)$ are vector spaces over $\mathbb{R}(s)$ with

$$
\operatorname{dim} \mathcal{N}_{r}(s)=n-r \text { and } \operatorname{dim} \mathcal{N}_{l}(s)=m-r
$$

It is known that $\mathcal{N}_{r}(s)$ and $\mathcal{N}_{l}(s)$ are spanned by minimal polynomial bases $\left\{\underline{x}_{i}(s)\right.$, $i=1,2, \ldots, n-r\}$ and $\left\{\underline{\psi}_{j}^{t}(s), i=1,2, \ldots, m-r\right\}$ of minimal degrees, namely (see $[5])$,

$$
\left\{v_{1}=v_{2}=\cdots=v_{g}=0<v_{g+1} \leq v_{g+2} \leq \cdots \leq v_{n-r}\right\}
$$

and

$$
\left\{u_{1}=u_{2}=\cdots=u_{h}=0<u_{h+1} \leq u_{h+2} \leq \cdots \leq u_{m-r}\right\}
$$


The sets of the minimal degrees $\left\{v_{i}, 1 \leq i \leq n-r\right\}$ and $\left\{u_{j}, 1 \leq j \leq m-r\right\}$ are known in [4] as column minimal indices (c.m.i.) and row minimal indices (r.m.i.) of $s F-G$, respectively. Furthermore, If $r=\operatorname{rank}_{\mathbb{R}(s)}(s F-G)<\min \{m, n\}$, then it is evident that

$$
r=\sum_{i=g+1}^{n-r} v_{i}+\sum_{j=h+1}^{m-r} u_{j}+\underset{\mathbb{R}(s)}{\operatorname{rank}}\left(s F_{w}-G_{w}\right),
$$

where $s F_{w}-G_{w}$ is the complex Weierstrass canonical form (see [4]) specified by the set of elementary divisors (e.d.) obtained by factoring the invariant polynomials $f(s, \hat{s})$ over $\mathbb{R}[s, \hat{s}]$ (the ring of polynomials in $s$ and $\hat{s}=1 / s$ with real coefficients), which are the nonzero elements on the diagonal of the Smith canonical form of the homogeneous pencil $s F-\hat{s} G$, into powers of homogeneous irreducible polynomials over $\mathbb{C}$.

Thus, in the case that $s F-G$ is a singular pencil, we have elementary divisors of the following types:

- e.d. of the type $s^{d}, d \in \mathbb{N}$, called zero finite elementary divisors (z. f.e.d.),

- e.d. of the type $(s-a)^{c}, a \neq 0, c \in \mathbb{N}$, called nonzero finite elementary divisors (nz. f.e.d.),

- e.d. of the type $\hat{s}^{q}$, called infinite elementary divisors (i.e.d),

- c.m.i. of the type $v \in \mathbb{N} \cup\{0\}$, called column minimal indices (c.m.i.) deduced from the column degrees of minimal polynomial bases of the maximal sub module $\mathcal{M}_{\mathcal{N}}$ embedded in $\mathcal{N}_{r}(s)$ with a free $\mathbb{R}(s)$-module structure,

- r.m.i. of the type $u \in \mathbb{N} \cup\{0\}$, called row minimal indices (r.m.i.) deduced from the row degrees of minimal polynomial bases of the maximal sub module $\mathcal{M}_{\mathcal{N}}$ embedded in $\mathcal{N}_{l}(s)$ with a free $\mathbb{R}(s)$-module structure.

For further details, see $[5,6,8]$.

Thus, there exist $P \in \mathbb{C}^{m \times m}$ and $Q \in \mathbb{C}^{n \times n}$ such that the complex Kronecker form of the singular pencil $s F-G$ is defined as

$$
\begin{aligned}
P(s F-G) Q & =s F_{k}-G_{k} \\
& \triangleq \text { block } \operatorname{diag}\left\{\mathbb{Q}_{h, g}, s \Lambda_{v}-\lambda_{v}, s \Lambda_{u}^{t}-\lambda_{u}^{t}, s I_{p}-J_{p}, s H_{q}-I_{q}\right\} .
\end{aligned}
$$

In more detail, matrix $\mathbb{Q}_{h, g}$ is uniquely defined by the sets $\underbrace{\{0,0, \ldots, 0\}}_{g}$ and $\underbrace{\{0,0, \ldots, 0\}}_{h}$ of zero column and row minimal indices, respectively.

The second normal block $s \Lambda_{v}-\lambda_{v}$ is uniquely defined by the set of nonzero column minimal indices (a new arrangement of the indices of $v$ must be noted in order to 
simplify the notation) $\left\{v_{g+1} \leq v_{g+2} \leq \cdots \leq v_{n-r}\right\}$ of $s F-G$ and has the form

$$
s \Lambda_{v}-\lambda_{v} \triangleq \text { block } \operatorname{diag}\left\{s \Lambda_{v_{g+1}}-\lambda_{v_{g+1}}, \ldots, s \Lambda_{v_{i}}-\lambda_{v_{i}}, \ldots, s \Lambda_{v_{n-r}}-\lambda_{v_{n-r}}\right\}
$$

where $\Lambda_{v_{i}}=\left[\begin{array}{ll}I_{v_{i}} & \underline{0}\end{array}\right] \in \mathbb{C}^{v_{i} \times\left(v_{i}+1\right)}, \lambda_{v_{i}}=\left[\begin{array}{ll}H_{v_{i}} & \underline{\varepsilon}_{v_{i}}\end{array}\right] \in \mathbb{C}^{v_{i} \times\left(v_{i}+1\right)}$ for every $i=g+$ $1, g+2, \ldots, n-r$, and $I_{v_{i}}$ and $H_{v_{i}}$ denote the $v_{i} \times v_{i}$ identity and the nilpotent (with annihilation index $v_{i}$ ) matrix, respectively. $\underline{0}$ and $\underline{\varepsilon}_{v_{i}}=\left[\begin{array}{llll}0 & \cdots & 0 & 1\end{array}\right]^{T} \in \mathbb{C}^{v_{i}}$ are the zero column and the column with element 1 in the last position, respectively.

The third normal block $s \Lambda_{u}^{t}-\lambda_{u}^{t}$ is uniquely determined by the set of nonzero row minimal indices (a new arrangement of the indices of $v$ must be noted in order to simplify the notation) $\left\{u_{h+1} \leq u_{h+2} \leq \cdots \leq u_{m-r}\right\}$ of $s F-G$ and has the form

$$
s \Lambda_{u}^{t}-\lambda_{u}^{t} \triangleq \text { block } \operatorname{diag}\left\{s \Lambda_{u_{h+1}}^{t}-\lambda_{u_{h+1}}^{t}, \ldots, s \Lambda_{u_{j}}^{t}-\lambda_{u_{j}}^{t}, \ldots, s \Lambda_{u_{m-r}}^{t}-\lambda_{u_{m-r}}^{t}\right\},
$$

where $\Lambda_{u_{j}}^{t}=\left[\begin{array}{c}\underline{e}_{u_{j}}^{t} \\ \cdots \\ H_{u_{j}}\end{array}\right] \in \mathbb{C}^{\left(u_{j}+1\right) \times u_{j}}, \lambda_{u_{j}}^{t}=\left[\begin{array}{c}\underline{0}^{t} \\ \cdots \\ I_{u_{j}}\end{array}\right] \in \mathbb{C}^{\left(u_{j}+1\right) \times u_{j}}$ for every $j=$ $h+1, h+2, \ldots, m-r$, and $I_{u_{j}}$ and $H_{u_{j}}$ denote the $u_{j} \times u_{j}$ identity and nilpotent (with annihilation index $u_{j}$ ) matrix and the zero column matrix, respectively. $\underline{0}$ and $\underline{e}_{u_{j}}=\left[\begin{array}{llll}1 & \cdots & 0 & 0\end{array}\right]^{T} \in \mathbb{C}^{u_{j}}$ are the zero column and the column with element 1 at the first position, respectively.

The forth and the fifth normal matrix block is the complex Weierstrass form $s F_{w}-G_{w}$ of the regular pencil $s F-G$, which is defined by

$$
s F_{w}-G_{w} \triangleq \text { block } \operatorname{diag}\left\{s I_{p}-J_{p}, s H_{q}-I_{q}\right\}
$$

the first normal Jordan type block $s I_{p}-J_{p}$ is uniquely defined by the set of f.e.d.

$$
\left(s-a_{i}\right)^{p_{i}}, \ldots,\left(s-a_{v}\right)^{p_{v}}, \sum_{j=1}^{v} p_{j}=p
$$

of $s F-G$ and has the form

$$
s I_{p}-J_{p} \triangleq \text { block } \operatorname{diag}\left\{s I_{p_{1}}-J_{p_{1}}\left(a_{1}\right), \ldots, s I_{p_{v}}-J_{p_{v}}\left(a_{v}\right)\right\} .
$$

Finally, the $q$ blocks of the second uniquely defined block $s H_{q}-I_{q}$ correspond to the i.e.d.

$$
(\hat{s})^{q_{1}}, \ldots,(\hat{s})^{q_{\sigma}}, \sum_{j=1}^{\sigma} q_{j}=q\left(q_{1} \leq q_{2} \leq \cdots \leq q_{\sigma}\right)
$$

of $s F-G$, having the form

$$
s H_{q}-I_{q} \triangleq \operatorname{block} \operatorname{diag}\left\{s H_{q_{1}}-I_{q_{1}}, \ldots, s H_{q_{\sigma}}-I_{q_{\sigma}}\right\} .
$$


Thus, $H_{q}$ is a nilpotent matrix of index $\tilde{q}=\max \left\{q_{j}: j=1,2, \ldots, \sigma\right\}$, where

$$
H_{q}^{\tilde{q}}=\mathbb{O} \text {. }
$$

The matrices $I_{p_{i}}, J_{p_{i}}\left(a_{i}\right), H_{q_{i}}$ are

$$
\begin{aligned}
& I_{p_{i}}=\left[\begin{array}{cccc}
1 & 0 & \cdots & 0 \\
0 & 1 & \cdots & 0 \\
\vdots & \vdots & \ddots & \vdots \\
0 & 0 & \cdots & 1
\end{array}\right] \in \mathbb{R}^{p_{i} \times p_{i}} \\
& J_{p_{i}}\left(a_{i}\right)=\left[\begin{array}{ccccc}
a & 1 & 0 & \cdots & 0 \\
0 & a & 1 & \cdots & 0 \\
\vdots & \vdots & \ddots & \vdots & \vdots \\
0 & 0 & 0 & a & 1 \\
0 & 0 & 0 & 0 & a
\end{array}\right] \in \mathbb{C}^{p_{i} \times p_{i}} \text {, and } H_{q_{i}}=\left[\begin{array}{ccccc}
0 & 1 & 0 & \cdots & 0 \\
0 & 0 & 1 & \cdots & 0 \\
\vdots & \vdots & \ddots & \vdots & \vdots \\
0 & 0 & 0 & 0 & 1 \\
0 & 0 & 0 & 0 & 0
\end{array}\right] \in \mathbb{R}^{q_{i} \times q_{i}}
\end{aligned}
$$

Definition 1.2. The dimension of a vector subspace is denoted by $\operatorname{dim}(\cdot)$. Given $A \in \mathbb{C}^{n \times n}$, the range (column space) of $A$ is denoted by $\Re(A)$; the null space of $A$, $\{\underline{x} \neq \underline{0}: A \underline{x}=\underline{0}\}$, is denoted by $\aleph(A)$. Recall that $\operatorname{dim} \aleph(A)+\operatorname{dim} \Re(A)=n$.

Definition 1.3. Let $A \in \mathbb{C}^{n \times n}$. The nonnegative integer $k$ is the index of $A$, denoted by $\operatorname{Ind}(A)=k$, if $k$ is the smallest nonnegative integer such that

$$
\operatorname{rank}\left(A^{k}\right)=\operatorname{rank}\left(A^{k+1}\right) .
$$

Definition 1.4. [3] The Moore-Penrose inverse of rectangular matrix $A \in \mathbb{C}^{m \times n}$ is the matrix $A^{\dagger} \in \mathbb{C}^{n \times n}$ that satisfies

(i) $A A^{\dagger} A=A$,

(ii) $A^{\dagger} A A^{\dagger}=A^{\dagger}$,

(iii) $\left(A A^{\dagger}\right)^{*}=A A^{\dagger}$,

(iv) $\left(A^{\dagger} A\right)^{*}=A^{\dagger} A$,

where ${ }^{*}$ denotes the conjugate transpose.

Moreover, the Drazin inverse of $A \in \mathbb{C}^{n \times n}$ with $\operatorname{Ind}(A)=k$ is the matrix $A^{D}$ satisfying

(i) $A^{D} A A^{D}=A^{D}$,

(ii) $A A^{D}=A^{D} A$,

(iii) $A^{l+1} A^{D}=A^{l}$ for $l \geq k=\operatorname{Ind}(A)$.

Note that if $A$ is nonsingular, then $A^{\dagger} \equiv A^{D} \equiv A^{-1}$. 
TheOREM 1.5. [11] For any square matrix $A \in \mathbb{C}^{n \times n}$ with $\operatorname{Ind}(A)=k$ and $\operatorname{rank}\left(A^{k}\right)=r$, there exists unique matrix $X$ of order $n$ such that

$$
A^{k} X=\mathbb{O}, \quad X A^{k}=\mathbb{O}, \quad X^{2}=X, \quad \operatorname{rank}(X)=n-r .
$$

For the above-mentioned matrix $X$, there exists a unique matrix $Y$ of order $n$ such that

$$
\operatorname{rank}\left[\begin{array}{cc}
A & I-X \\
I-X & Y
\end{array}\right]=\operatorname{rank}(A)
$$

The matrix $Y$ is the Drazin inverse $A^{D}$ of $A$ and $X=I-A A^{D}$.

Theorem 1.6. [11] Consider a square matrix $A \in \mathbb{C}^{n \times n}$ with $\operatorname{rank}(A)=r \geq 1$. Let $A[a \mid b]$ be the $r \times r$ nonsingular submatrix of $A$ which has row index set $a=$ $\left\{i_{1}, i_{2}, \ldots, i_{r}\right\}$ and the column index set $b=\left\{j_{1}, j_{2}, \ldots, j_{r}\right\}$. If the matrix $X$ satisfies condition (1.19), then we have

$$
A^{D}=(I-X)[N \mid a](A[a \mid b])^{-1}(I-X)[b \mid N],
$$

where $N=\{1,2, \ldots, n\}$.

A theorem and a corollary characterizing the Moore-Penrose inverse are Theorem 1 in $[7$, p. 130] and its corollary in [7, p. 132].

2. Main results for rectangular coefficient matrices. In this section we shall consider two important cases with many practical applications. First of all, we investigate the relation between the Drazin inverse and the matrix pencil approach. If $\mu F+G$ is one-to-one (i.e. full column rank - only row minimal indices) for some $\mu \in \mathbb{C}$, then $m>n$ and the system is over-determined (i.e. unique solution). If $\mu F+G$ is onto (i.e. full row rank - only column minimal indices) for some $\mu \in \mathbb{C}$, then $m<n$ and the system is under-determined (i.e. the solution always exists but it is not unique).

Definition 2.1. Let $F, G \in \mathbb{C}^{m \times n}$

a) When $\mu F+G$ is one-to-one, we define the $n$-square matrices

$$
\hat{F}_{\mu} \triangleq(\mu F+G)^{\dagger} F \in \mathbb{C}^{n \times n} \text { and } \hat{G}_{\mu} \triangleq(\mu F+G)^{\dagger} G \in \mathbb{C}^{n \times n} .
$$

b) When $\mu F+G$ is onto, we define the $m$-square matrices

$$
\hat{F}_{\mu} \triangleq(\mu F+G)^{\dagger} F \in \mathbb{C}^{m \times m} \text { and } \hat{G}_{\mu} \triangleq(\mu F+G)^{\dagger} G \in \mathbb{C}^{m \times m} .
$$

The following known result is very important for the entire discussion.

Lemma 2.2. [5] Let $F, G \in \mathbb{C}^{m \times n}$. 
a) If $\mu F+G$ is one-to-one (i.e. full column rank and $r=\operatorname{rank}(\mu F+G)=$ $\left.\sum_{j=h+1}^{m-n} u_{j}=n<m\right)$ for some $\mu \in \mathbb{C}$ (or similarly $\left.\mathcal{N}_{r}(\mu)=\{\underline{0}\}\right)$, then there exist $P \in \mathbb{C}^{m \times m}$ and $Q \in \mathbb{C}^{n \times n}$ such as the complex Kronecker form $\mu F_{k}+G_{k}$ of the pencil $\mu F+G$ is given by

$$
\begin{aligned}
& P(\mu F+G) Q=\mu F_{k}+G_{k} \\
& \triangleq \text { block diag }\left\{\left[\begin{array}{c}
\mathbb{O}_{h, u_{h+1}} \\
\mu \Lambda_{u_{h+1}}^{t}+\lambda_{u_{h+1}}^{t}
\end{array}\right], \mu \Lambda_{u_{h+2}}^{t}+\lambda_{u_{h+2}}^{t}, \ldots, \mu \Lambda_{u_{m-n}}^{t}+\lambda_{u_{m-n}}^{t}\right\},
\end{aligned}
$$

where the blocks $\mu \Lambda_{u_{j}}^{t}+\lambda_{u_{j}}^{t}$ for $j=h+1, h+2, \ldots, m-n$ are uniquely determined by the set of nonzero row minimal indices; see expression (1.11) and Section 1 for more details.

b) If $\mu F+G$ is onto (i.e. full row rank and $r=\operatorname{rank}(\mu F+G)=\sum_{j=g+1}^{n-m} v_{j}=$ $m<n$ ) for some $\mu \in \mathbb{C}$ (or similarly $\mathcal{N}_{l}(\mu)=\left\{\underline{0}^{t}\right\}$ ), then there exist $P \in \mathbb{C}^{m \times m}$ and $Q \in \mathbb{C}^{n \times n}$ such as the complex Kronecker form of the pencil $\mu F+G$ is given by

$$
\begin{aligned}
P(\mu F+G) Q=\mu F_{k}+G_{k}= & \text { block } \operatorname{diag}\left\{\left[\mathbb{O}_{v_{g+1}, g} \mu \Lambda_{v_{g+1}}+\lambda_{v_{g+1}}\right]\right. \\
& \left.\mu \Lambda v_{g+2}+\lambda_{v_{g+2}}, \ldots, \mu \Lambda_{v_{n-m}}+\lambda_{v_{n-m}}\right\}
\end{aligned}
$$

where the blocks $\mu \Lambda_{v_{i}}+\lambda_{v_{i}}$ for $i=g+1, g+2, \ldots, n-m$ are uniquely defined by the set of nonzero column minimal indices; see expression (1.10) and Section 1 for more details.

REMARK 2.3. In this part of the paper, we should stress out that the matrix $\left[\begin{array}{c}\underline{e}^{t} \\ \cdots \\ H_{u_{j}}\end{array}\right]$ can also be written as $\left[\begin{array}{c}I_{u_{h+1}} \\ \cdots \\ \underline{0}^{t}\end{array}\right] \in \mathbb{C}^{\left(u_{j}+1\right) \times u_{j}}$. In the same sense, the matrix $\left[\begin{array}{ll}H_{v_{i}} & \underline{\varepsilon}_{v_{i}}\end{array}\right]$ can also be expressed as $\left[\underline{0} I_{v_{i}}\right] \in \mathbb{C}^{v_{i} \times\left(v_{i}+1\right)}$. As we may see in the following lemmas, Remark 2.1 is very useful indeed.

Lemma 2.4. For the identity matrix $I_{p} \in \mathbb{C}^{p \times p}$, the nilpotent matrix $H_{p} \in \mathbb{C}^{p \times p}$ (with annihilation index $p$ ), the column vector $\underline{e}_{u_{j}} \in \mathbb{C}^{u_{j}}$ for $j=h+1, h+2, \ldots, m-r$ (with element 1 at the first place), and $\underline{\varepsilon}_{v_{i}} \in \mathbb{C}^{v_{i}}$ for $i=g+1, g+2, \ldots, n-r$ (with element 1 at the $v_{i}$-place) and $\mu \in \mathbb{C} \backslash\{0\}$, we have that

i)

$$
\begin{array}{r}
{\left[\begin{array}{c}
\mu \underline{e}_{u_{j}}^{t} \\
\cdots \\
\mu H_{u_{j}}+I_{u_{j}}
\end{array}\right]^{\dagger}=\left[(\bar{\mu} \mu+1) I_{u_{j}}+\mu H_{u_{j}}+\bar{\mu} H_{u_{j}}^{t}\right]^{-1}} \\
{\left[\bar{\mu} I_{u_{j}}+H_{u_{j}} \underline{\varepsilon}_{u_{j}}\right] \in \mathbb{C}^{u_{j} \times\left(u_{j}+1\right)},}
\end{array}
$$


ii)

$$
\begin{aligned}
{\left[\mu I_{v_{i}}+H_{v_{i}} \vdots \underline{\varepsilon}_{v_{i}}\right]^{\dagger}=} & {\left[\begin{array}{c}
\bar{\mu} I_{v_{i}}+H_{v_{i}}^{t} \\
\underline{\varepsilon}_{v_{i}}^{t}
\end{array}\right] } \\
& {\left[(\bar{\mu} \mu+1) I_{v_{i}}+\bar{\mu} H_{v_{i}}+\mu H_{v_{i}}^{t}\right]^{-1} \in \mathbb{C}^{\left(v_{i}+1\right) \times v_{i}} . }
\end{aligned}
$$

Proof. i) Consider the block matrix

$$
A \triangleq\left[\begin{array}{c}
\mu \underline{e}_{u_{j}}^{t} \\
\cdots \\
\mu H_{u_{j}}+I_{u_{j}}
\end{array}\right]=\left[\begin{array}{ccccc}
\mu & 0 & 0 & \cdots & 0 \\
1 & \mu & 0 & \cdots & 0 \\
0 & 1 & \mu & \cdots & 0 \\
\vdots & \vdots & \vdots & \ddots & \vdots \\
0 & 0 & 0 & \cdots & \mu \\
0 & 0 & 0 & \cdots & 1
\end{array}\right] \in \mathbb{C}^{\left(u_{j}+1\right) \times u_{j}}
$$

Now, by Theorem 1.3.2 of [2], the Moore-Penrose inverse matrix of $A$ is given by

$$
A^{\dagger}=\left(A^{*} A\right)^{-1} A^{*}
$$

Finally, since $A^{*}=\left[\begin{array}{ccccc}\bar{\mu} & 1 & 0 & \cdots & 0 \\ 0 & \bar{\mu} & 1 & \cdots & 0 \\ 0 & 0 & \bar{\mu} & \cdots & 0 \\ \vdots & \vdots & \vdots & \ddots & \vdots \\ 0 & 0 & 0 & \cdots & 0 \\ 0 & 0 & 0 & \cdots & 1\end{array}\right]=\left[\bar{\mu} I_{u_{j}}+H_{u_{j}} \quad \varepsilon_{u_{j}}\right] \in \mathbb{C}^{u_{j} \times\left(u_{j}+1\right)}$,

$$
\begin{aligned}
A^{*} A & =\left[\begin{array}{cccccc}
\bar{\mu} \mu+1 & \mu & 0 & \cdots & 0 & 0 \\
\bar{\mu} & \bar{\mu} \mu+1 & \mu & \cdots & 0 & 0 \\
0 & \bar{\mu} & \bar{\mu} \mu+1 & \cdots & 0 & 0 \\
\vdots & \vdots & \vdots & \ddots & \vdots & \vdots \\
0 & 0 & 0 & \cdots & \bar{\mu} \mu+1 & \mu \\
0 & 0 & 0 & \cdots & \bar{\mu} & \bar{\mu} \mu+1
\end{array}\right] \\
& =\left[(\bar{\mu} \mu+1) I_{u_{j}}+\mu H_{u_{j}}+\bar{\mu} H_{u_{j}}^{t}\right]
\end{aligned}
$$

where the $\bar{\mu} \in \mathbb{C}$ is the conjugate of $\mu$. Expression (2.3) is proven. 
ii) Consider the block matrix

$$
B \triangleq\left[\mu I_{v_{i}}+H_{v_{i}} \quad \underline{e}_{v_{i}}\right]=\left[\begin{array}{ccccc}
\mu & 1 & 0 & \cdots & 0 \\
0 & \mu & 1 & \cdots & 0 \\
0 & 0 & \mu & \cdots & 0 \\
\vdots & \vdots & \vdots & \ddots & \vdots \\
0 & 0 & 0 & \cdots & 0 \\
0 & 0 & 0 & \cdots & 1
\end{array}\right] \in \mathbb{C}^{v_{i} \times\left(v_{i}+1\right)} .
$$

Again by Theorem 1.3.2 of [2], the Moore-Penrose inverse matrix of $A$ is given by

$$
\begin{aligned}
\text { Finally, since } B^{*}= & {\left[\begin{array}{ccccc}
\bar{\mu} & 0 & 0 & \cdots & 0 \\
1 & \bar{\mu} & 0 & \cdots & 0 \\
0 & 1 & \bar{\mu} & \cdots & 0 \\
\vdots & \vdots & \vdots & \ddots & \vdots \\
0 & 0 & 0 & \cdots & \bar{\mu} \\
0 & 0 & 0 & \cdots & 1
\end{array}\right]=\left[\begin{array}{c}
\bar{\mu} I_{v_{i}}+H_{v_{i}}^{t} \\
\underline{\varepsilon}_{v_{i}}^{t}
\end{array}\right] \in \mathbb{C}^{\left(v_{i}+1\right) \times v_{i}}, } \\
B B^{*} & =\left[\begin{array}{cccccc}
\bar{\mu} \mu+1 & \bar{\mu} & 0 & \cdots & 0 & 0 \\
\mu & \bar{\mu} \mu+1 & \bar{\mu} & \cdots & 0 & 0 \\
0 & \mu & \bar{\mu} \mu+1 & \cdots & 0 & 0 \\
\vdots & \vdots & \vdots & \ddots & \vdots & \vdots \\
0 & 0 & 0 & \cdots & \bar{\mu} \mu+1 & \bar{\mu} \\
0 & 0 & 0 & \cdots & \mu & \bar{\mu} \mu+1
\end{array}\right] \\
& =\left[(\bar{\mu} \mu+1) I_{v_{i}}+\bar{\mu} H_{v_{i}}+\mu H_{v_{i}}^{t}\right] \in \mathbb{C}^{v_{i} \times v_{i}},
\end{aligned}
$$

where the $\bar{\mu} \in \mathbb{C}$ is the conjugate of $\mu$. Expression (2.4) is proven. $\square$

Theorem 2.5. Consider matrices $\hat{F}_{\mu} \in \mathbb{C}^{n \times n}$ and $\hat{G}_{\mu} \in \mathbb{C}^{m \times m}$.

a) If $\mu F+G$ is one-to-one, then

$$
\hat{F}_{\mu}=Q\left[\begin{array}{c}
{\left[(\bar{\mu} \mu+1) I_{u_{h+1}}+\mu H_{u_{h+1}}+\bar{\mu} H_{u_{h+1}}^{t}\right]^{-1}\left(\bar{\mu} I_{u_{h+1}}+H_{u_{h+1}}\right)} \\
\ddots \\
{\left[(\bar{\mu} \mu+1) I_{u_{m-n}}+\mu H_{u_{m-n}}+\bar{\mu} H_{u_{m-n}}^{t}\right]^{-1}\left(\bar{\mu} I_{u_{m-n}}+H_{u_{m-n}}\right)}
\end{array}\right] Q^{-1}
$$

and $\hat{G}_{\mu}=I_{n}-\mu \hat{F}_{\mu}$.

b) If $\mu F+G$ is onto, then

$$
\hat{F}_{\mu}=P^{-1}\left[\begin{array}{c}
\left(\bar{\mu} I_{v_{g+1}}+H_{v_{g+1}}^{t}\right)\left[(\bar{\mu} \mu+1) I_{u_{g+1}}+\bar{\mu} H_{u_{g+1}}+\mu H_{u_{g+1}}^{t}\right]^{-1} \\
\ddots \\
\ddots \\
\left(\bar{\mu} I_{v_{n-m}}+H_{v_{n-m}}^{t}\right)\left[(\bar{\mu}+1) I_{u_{n-m}}+\bar{\mu} H_{u_{n-m}}+\mu H_{u_{n-m}}^{t}\right]^{-1}
\end{array}\right] P
$$


and $\hat{G}_{\mu}=I_{m}-\mu \hat{F}_{\mu}$.

Proof. Following Definition 2.1 (a) and Lemma 2.2, there always exists $\mu \in \mathbb{C}$ such that the matrix $(\mu F+G)$ is one-to-one for rectangular matrices $F, G \in \mathbb{C}^{m \times n}$.

a) Now, multiplying on the left with $(\mu F+G)^{\dagger}$ the system (1.1) (analogously, the system (1.2)), we obtain

$$
(\mu F+G)^{\dagger} F \underline{x}^{\prime}(t)=(\mu F+G)^{\dagger} G \underline{x}(t) \stackrel{D 2.1}{\Longleftrightarrow} \hat{F}_{\mu} \underline{x}^{\prime}(t)=\hat{G}_{\mu} \underline{x}(t) .
$$

Hence, there exist invertible matrices $P \in \mathbb{C}^{m \times m}, Q \in \mathbb{C}^{n \times n}$ giving the complex Kronecker canonical form; see (2.1) (or (2.2)). Consequently,

$$
\begin{aligned}
\hat{F}_{\mu}=(\mu F+G)^{\dagger} F & =\left(\mu P^{-1} F_{k} Q^{-1}+P^{-1} G_{k} Q^{-1}\right)^{\dagger} P^{-1} F_{k} Q^{-1} \\
& =Q\left(\mu F_{k}+G_{k}\right)^{\dagger} F_{k} Q^{-1}
\end{aligned}
$$

and

$$
\begin{aligned}
\hat{G}_{\mu}=(\mu F+G)^{\dagger} G & =\left(\mu P^{-1} F_{k} Q^{-1}+P^{-1} G_{k} Q^{-1}\right)^{\dagger} P^{-1} G_{k} Q^{-1} \\
& =Q\left(\mu F_{k}+G_{k}\right)^{\dagger} G_{k} Q^{-1} .
\end{aligned}
$$

Moreover, using expressions (1.11) and (2.1), we obtain

$$
\begin{aligned}
& \left(\mu F_{k}+G_{k}\right)^{\dagger} \\
& =\left[\begin{array}{lllll}
\mathbb{Q}_{u_{h+1}, h} & \left(\mu \Lambda_{u_{h+1}}^{t}+\lambda_{u_{h+1}}^{t}\right)^{\dagger} & & \\
& \left(\mu \Lambda_{u_{h+2}}^{t}+\lambda_{u_{h+2}}^{t}\right)^{\dagger} & & \\
& & \ddots & \\
& & \left(\mu \Lambda_{u_{m-n}}^{t}+\lambda_{u_{m-n}}^{t}\right)^{\dagger}
\end{array}\right]
\end{aligned}
$$

Consequently, substituting the above expression into (2.9), expression (2.1) follows, that is,

$$
\begin{aligned}
& \left(\mu F_{k}+G_{k}\right)^{\dagger} F_{k} \\
& =\left[\begin{array}{llll}
\left(\mu \Lambda_{u_{h+1}}^{t}+\lambda_{u_{h+1}}^{t}\right)^{\dagger} \Lambda_{u_{h+1}}^{t} & & \\
& \left(\mu \Lambda_{u_{h+2}}^{t}+\lambda_{u_{h+2}}^{t}\right)^{\dagger} \Lambda_{u_{h+2}}^{t} & & \\
& & \ddots & \\
& & \left(\mu \Lambda_{u_{m-n}}^{t}+\lambda_{u_{m-n}}^{t}\right)^{\dagger} \Lambda_{u_{m-n}}^{t}
\end{array}\right]
\end{aligned}
$$




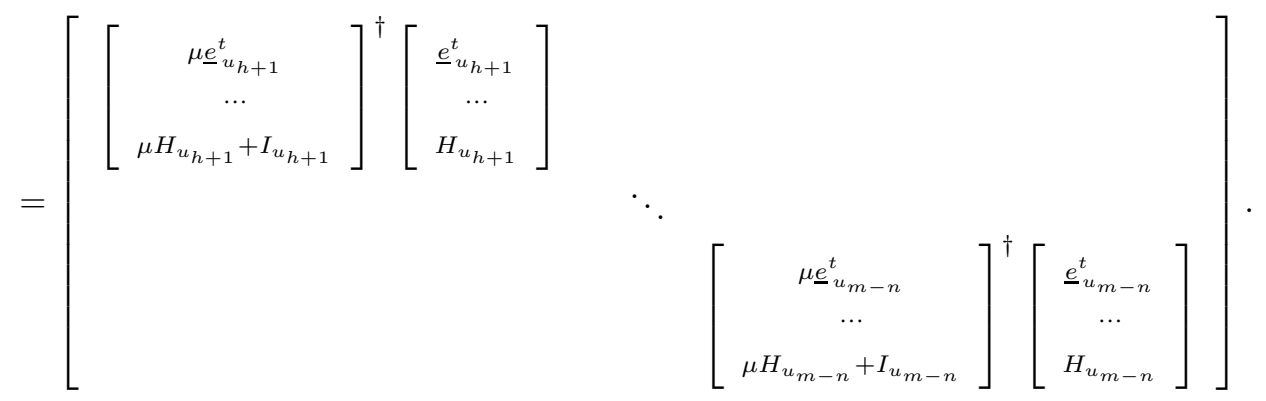

Considering Lemma 2.2, we obtain

$$
\begin{aligned}
{\left[\begin{array}{c}
\mu \underline{e}_{u_{j}}^{t} \\
\ldots \\
\mu H_{u_{j}}+I_{u_{j}}
\end{array}\right]^{\dagger}\left[\begin{array}{c}
\underline{e}_{u_{j}} \\
\cdots \\
H_{u_{j}}
\end{array}\right] } & =\left[(\bar{\mu} \mu+1) I_{u_{j}}+\mu H_{u_{j}}+\bar{\mu} H_{u_{j}}^{t}\right]^{-1}\left[\bar{\mu} I_{u_{j}}+H_{u_{j}} \underline{\varepsilon}_{u_{j}}\right]\left[\begin{array}{c}
\underline{\varepsilon}_{u_{j}} \\
\ldots \\
H_{u_{j}}
\end{array}\right] \\
& =\left[(\bar{\mu} \mu+1) I_{u_{j}}+\mu H_{u_{j}}+\bar{\mu} H_{u_{j}}^{t}\right]^{-1}\left(\bar{\mu} I_{u_{j}}+H_{u_{j}}\right) \in \mathbb{C}^{u_{j} \times u_{j}} .
\end{aligned}
$$

Note also that $\mu \hat{F}_{\mu}+\hat{G}_{\mu}=I_{n} \Rightarrow \hat{G}_{\mu}=I_{n}-\mu \hat{F}_{\mu}$, because $\mu \hat{F}_{\mu}+\hat{G}_{\mu}=\mu(\mu F+G)^{\dagger} F+$ $(\mu F+G)^{\dagger} G=(\mu F+G)^{\dagger}(\mu F+G)=I$.

b) Following Definition 2.1 (b) and Lemma 2.2, there always exists $\mu \in \mathbb{C}$ such that the matrix $(\mu F+G)$ is onto for rectangular matrices $F, G \in \mathbb{C}^{m \times n}$.

Here, defining $P_{\mu}=(\mu F+G)^{\dagger}(\mu F+G)$, the system (1.1) (analogously, the system (1.2)) gets transformed to

$$
F P_{\mu} \underline{x}^{\prime}(t)+F\left(I_{n}-P_{\mu}\right) \underline{x}^{\prime}(t)=G P_{\mu} \underline{x}(t)+G\left(I_{n}-P_{\mu}\right) \underline{x}(t),
$$

or equivalently,

$$
\begin{aligned}
& F(\mu F+G)^{\dagger}\left[(\mu F+G) \underline{x}^{\prime}(t)\right]+F\left(I_{n}-P_{\mu}\right) \underline{x}(t) \\
&=G(\mu F+G)^{\dagger}[(\mu F+G) \underline{x}(t)]+G\left(I_{n}-P_{\mu}\right) \underline{x}(t) \\
& \stackrel{D 2.1}{\Longleftrightarrow} \hat{F}_{\mu}\left[(\mu F+G) \underline{x}^{\prime}(t)\right]+F\left(I_{n}-P_{\mu}\right) \underline{x}^{\prime}(t)=\hat{G}_{\mu}[(\mu F+G) \underline{x}(t)]+G\left(I_{n}-P_{m}\right) \underline{x}(t) .
\end{aligned}
$$

Since $\mu \hat{F}_{\mu}+\hat{G}_{\mu}=I_{m}$, the system above is, in terms of $(\mu F+G) \underline{x}(t)$, a differential equation of the type already solved and hence has a solution for any choice of $\left(I_{m}-\right.$ $\left.P_{\mu}\right) \underline{x}(t)$.

Hence, there exist invertible matrices $P \in \mathbb{C}^{m \times m}, Q \in \mathbb{C}^{n \times n}$ giving the complex Kronecker canonical form; see (2.1) (or (2.2)). Consequently,

$$
\begin{aligned}
\hat{F}_{\mu}=F(\mu F+G)^{\dagger} & =P^{-1} F_{k} Q^{-1}\left(\mu P^{-1} F_{k} Q^{-1}+P^{-1} G_{k} Q^{-1}\right)^{\dagger} \\
& =P^{-1} F_{k}\left(\mu F_{k}+G_{k}\right)^{\dagger} P
\end{aligned}
$$


and

$$
\begin{aligned}
\hat{G}_{\mu}=G(\mu F+G)^{\dagger} & =P^{-1} G_{k} Q^{-1}\left(\mu P^{-1} F_{k} Q^{-1}+P^{-1} G_{k} Q^{-1}\right)^{\dagger} \\
& =P^{-1} G_{k}\left(\mu F_{k}+G_{k}\right)^{\dagger} P .
\end{aligned}
$$

Furthermore, using expressions (1.10) and (2.2)

$$
\left(\mu F_{k}+G_{k}\right)^{\dagger}=\left[\begin{array}{cccc}
\mathcal{O}_{g, v_{g+1}}^{\mathbb{O}_{\left.v_{g+1}+\lambda_{v_{g+1}}\right)^{\dagger}}} & & & \\
& \left(\mu \Lambda_{v_{g+2}}+\lambda_{v_{g+2}}\right)^{\dagger} & & \\
& & \ddots & \\
& & \left(\mu \Lambda_{v_{n-m}}+\lambda_{v_{n-m}}\right)^{\dagger}
\end{array}\right]
$$

Consequently, substituting the above expression into (2.12), expression (2.2) follows, that is,

$$
\begin{aligned}
& F_{k}\left(\mu F_{k}+G_{k}\right)^{\dagger} \\
& =\left[\begin{array}{llll}
\Lambda_{v_{g+1}}\left(\mu \Lambda_{v_{g+1}}+\lambda_{v_{g+1}}\right)^{\dagger} & & & \\
& \Lambda_{v_{g+2}}\left(\mu \Lambda_{v_{g+2}}+\lambda_{v_{g+2}}\right)^{\dagger} & & \\
& & \ddots & \\
& & \Lambda_{v_{n-m}}\left(\mu \Lambda_{v_{n-m}}+\lambda_{v_{n-m}}\right)^{\dagger}
\end{array}\right]
\end{aligned}
$$

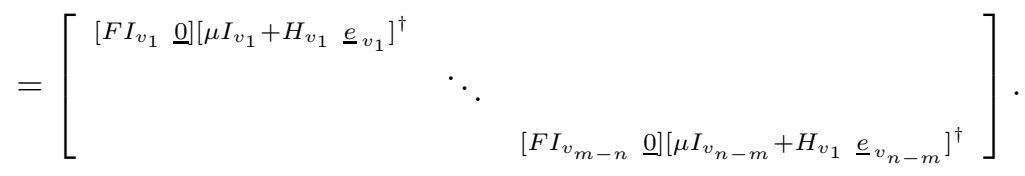

Considering Lemma 2.4, we obtain

$$
\begin{aligned}
& {\left[\begin{array}{ll}
\left.I_{v_{1}} \underline{0}\right]\left[\mu I_{v_{1}}+H_{v_{1}} \underline{e}_{v_{1}}\right. & ]^{\dagger}
\end{array}=\left[\begin{array}{ll}
I_{v_{1}} & \underline{0}
\end{array}\right]\left[\begin{array}{c}
\bar{\mu} I_{v_{1}}+H_{v_{1}}^{t} \\
\underline{\varepsilon}_{v_{1}}^{t}
\end{array}\right]\left[(\bar{\mu} \mu+1) I_{v_{1}}+\bar{\mu} H_{v_{1}}+\mu H_{v_{1}}^{t}\right]^{-1}\right.} \\
&=\left(\bar{\mu} I_{v_{1}}+H_{v_{1}}^{t}\right)\left[(\bar{\mu} \mu+1) I_{v_{1}}+\bar{\mu} H_{v_{1}}+\mu H_{v_{1}}^{t}\right]^{-1} \in \mathbb{C}^{v_{i} \times v_{i}} .
\end{aligned}
$$

Note the formulas above can be simplified considerably if $F$ or $G$ are onto. In applications, the case when $G$ is onto is more important. Moreover, we can easily verify that $\mu \hat{F}_{\mu}+\hat{G}_{\mu}=I_{m} \Rightarrow \hat{G}_{\mu}=I_{m}-\mu \hat{F}_{\mu}$, because $\mu \hat{F}_{\mu}+\hat{G}_{\mu}=\mu F(\mu F+G)^{\dagger}+G(\mu F+G)^{\dagger}=$ $(\mu F+G)(\mu F+G)^{\dagger}=I_{m}$.

Corollary 2.6. Matrices $\hat{F}_{\mu} \in \mathbb{C}^{n \times n}$ and $\hat{F}_{\mu} \in \mathbb{C}^{m \times m}$ are invertible and their inverses, respectively, are 
a)

$$
\hat{F}_{\mu}^{-1}=Q\left[\begin{array}{c}
\left(\mu I_{u_{h+1}}+H_{u_{h+1}}\right)^{-1}\left[(\bar{\mu} \mu+1) I_{u_{h+1}}+\mu H_{u_{h+1}}+\bar{\mu} H_{u_{h+1}}^{t}\right] \\
\ddots \\
\underbrace{}_{\left(\mu I_{u_{m-n}}+H_{u_{m-n}}\right)^{-1}\left[(\bar{\mu} \mu+1) I_{u_{m-n}}+\mu H_{u_{m-n}}+\bar{\mu} H_{u_{m-n}}^{t}\right]}
\end{array}\right] Q^{-1}
$$

b)

$$
\hat{F}_{\mu}^{-1}=P^{-1}\left[\begin{array}{c}
{\left[(\bar{\mu} \mu+1) I_{u_{g+1}}+\bar{\mu} H_{u_{g+1}}+\mu H_{u_{g+1}}^{t}\right]\left(\bar{\mu} I_{v_{g+1}}+H_{v_{g+1}}^{t}\right)^{-1}} \\
\ddots \\
{\left[(\bar{\mu} \mu+1) I_{u_{n-m}}+\bar{\mu} H_{u_{n-m}}+\mu H_{u_{n-m}}^{t}\right]\left(\bar{\mu} I_{v_{n-m}}+H_{v_{n-m}}^{t}\right)^{-1}}
\end{array}\right] P .
$$

Proof. Since the proofs of a) and b) are similar, only the proof for b) is presented.

b) The square matrix $P \hat{F}_{\mu} P^{-1} \in \mathbb{C}^{m \times m} \operatorname{has} \operatorname{rank}\left(P \hat{F}_{\mu} P^{-1}\right)=\operatorname{rank}\left(\hat{F}_{\mu}\right)=m \geq$ 1. According to Theorem 1.6, since $P \hat{F}_{\mu} P^{-1}$ is a $m \times m$ nonsingular matrix, the unique matrix $X$ of order $m$ that satisfies conditions (1.19) is $X=\mathbb{O}_{m}$. In order to prove expression (2.14), we should also consider that

$$
\left(P \hat{F}_{\mu} P^{-1}\right)^{D}=P \hat{F}_{\mu}^{D} P^{-1}
$$

Since the proof of (2.17) is a straightforward application of the (Drazin) inverse definition, we finally obtain the required expression.

ExAMPLE 2.7. Consider the system (1.1) (analogously with system (1.2))

$$
F \underline{x}^{\prime}(t)=G \underline{x}(t)
$$

with $F=\left[\begin{array}{rrrr}0 & 1 & -1 & 0 \\ 0 & 0 & 1 & 0\end{array}\right]$ and $G=\left[\begin{array}{rrrr}0 & 0 & 1 & -1 \\ 0 & 0 & 0 & 1\end{array}\right]$.

If we set $\mu=1, F+G=\left[\begin{array}{rrrr}0 & 1 & 0 & -1 \\ 0 & 0 & 1 & 1\end{array}\right]$ is onto with $\operatorname{rank}(F+G)=v_{2}=2$. Then, there exist $P=\left[\begin{array}{ll}1 & 1 \\ 0 & 1\end{array}\right]$ and $Q=I_{4}$ such that

$$
P(F+G) Q=\left[\begin{array}{llll}
0 & 1 & 1 & 0 \\
0 & 0 & 1 & 1
\end{array}\right]
$$


Using Lemma 2.3 (b) and (2.13), we obtain

$$
\begin{aligned}
& (F+G)^{\dagger}=Q\left(F_{k}+G_{k}\right)^{\dagger} P=Q\left[\begin{array}{c}
\underline{0}^{t} \\
{\left[I_{v_{2}}+H_{v_{2}} \underline{e}_{v_{2}}\right]^{\dagger}}
\end{array}\right] P \\
& =Q\left[\begin{array}{c}
I_{v_{2}}+H_{v_{2}}^{t} \\
\underline{\varepsilon}_{v_{2}}^{t}
\end{array}\right]\left[\begin{array}{c}
\underline{0}^{t} \\
{\left[2 I_{v_{2}}+H_{v_{2}}+H_{v_{2}}^{t}\right]^{-1}}
\end{array}\right] P \\
& =\frac{1}{3} I_{4}\left[\begin{array}{rr}
0 & 0 \\
2 & -1 \\
1 & 1 \\
-1 & 2
\end{array}\right]\left[\begin{array}{ll}
1 & 1 \\
0 & 1
\end{array}\right] P=\frac{1}{3}\left[\begin{array}{rr}
0 & 0 \\
2 & 1 \\
1 & 2 \\
-1 & 1
\end{array}\right] \text {, } \\
& P_{1}=(F+G)^{\dagger}(F+G)=\frac{1}{3}\left[\begin{array}{cccc}
0 & 0 & 0 & 0 \\
0 & 2 & 1 & 1 \\
0 & 1 & 2 & 1 \\
0 & 1 & 1 & 2
\end{array}\right] \\
& \hat{F}_{1}^{D} \equiv \hat{F}_{1}^{-1}=P^{-1}\left[2 I_{v_{2}}+H_{v_{2}}+H_{v_{2}}^{t}\right]\left(I_{v_{2}}+H_{v_{2}}^{t}\right)^{-1} P=\left[\begin{array}{rr}
2 & 1 \\
-1 & 1
\end{array}\right], \\
& \hat{F}_{1}=P^{-1}\left(I_{v_{2}}+H_{v_{2}}^{t}\right)\left[2 I_{v_{2}}+H_{v_{2}}+H_{v_{2}}^{t}\right]^{-1} P=\left[\begin{array}{rr}
1 & -1 \\
1 & 2
\end{array}\right],
\end{aligned}
$$

and $\hat{G}_{1}=I_{2}-\hat{F}_{1}=\left[\begin{array}{rr}0 & 1 \\ -1 & -1\end{array}\right]$, respectively. Thus, the solution (see e.g., $[2,3]$ ) is given by

$$
\begin{aligned}
\underline{x} & =(F+G)^{\dagger} e^{-\hat{F}_{1}^{D} \hat{G}_{1} t} \hat{F}_{1} \hat{F}_{1}^{D} \underline{q}+\left[I_{4}-P_{1}\right] \underline{h} \\
& =\left[\begin{array}{rr}
0 & 0 \\
2 & 1 \\
1 & 2 \\
-1 & 1
\end{array}\right] e^{-\left[\begin{array}{rr}
-1 & 1 \\
-1 & -2
\end{array}\right] t} \underline{t}-\frac{1}{3}\left[\begin{array}{rrrr}
-3 & 0 & 0 & 0 \\
0 & -1 & 1 & 1 \\
0 & 1 & -1 & 1 \\
0 & 1 & 1 & -1
\end{array}\right] \underline{h}
\end{aligned}
$$

for arbitrary $\underline{q} \in \mathbb{C}^{2}$ and function $\underline{h} \in \mathbb{C}^{4}$.

3. The Drazin inverse for square matrices. In this section, we revisit the recent results of [9], in order to provide a more algorithmic method for the calculation of the Drazin inverse of $\hat{F}_{\mu}$ (and $F$ ) through the Weierstrass canonical form in the sense of [11]. More analytically, let us firstly introduce the following definition and lemma. 
Definition 3.1. Define the matrices $\hat{F}_{\mu} \triangleq(\mu F+G)^{-1} F$ and $\hat{G}_{\mu} \triangleq(\mu F+G)^{-1} G$, for $\mu \in \mathbb{C}$, where $F, G \in \mathbb{C}^{n \times n}$.

Lemma 3.2. [9] For matrices $I_{p} \in \mathbb{R}^{p \times p}, J_{p}\left(\lambda_{j}\right) \in \mathbb{C}^{p \times p}$, and $H_{q} \in \mathbb{C}^{q \times q}$, it follows that matrices $\hat{F}_{\mu}$ and $\hat{G}_{\mu}$ satisfy

$$
Q^{-1} \hat{F}_{\mu} Q=\left[\begin{array}{cc}
\left(\mu I_{p}+J_{p}\right)^{-1} & O_{p, q} \\
O_{q, p} & \left(\mu H_{q}+I_{q}\right)^{-1} H_{q}
\end{array}\right],
$$

and $\hat{G}_{\mu}=I-\mu \hat{F}_{\mu}$, respectively.

The algorithmic approach for the calculation of the Drazin inverse of matrix $\hat{F}_{\mu}$ (and $F$ ) is derived via Proposition 3.3 (and Proposition 3.4, respectively).

Proposition 3.3. The Drazin inverse of $\hat{F}_{\mu}$ is given by

$$
\hat{F}_{\mu}^{D}=Q\left[\begin{array}{cc}
\mu I_{p}+J_{p} & O_{p, q} \\
O_{q, p} & O_{q}
\end{array}\right] Q^{-1}
$$

Proof. Considering (3.1), we can easily verify that

$$
\operatorname{rank}\left(Q \hat{F}_{\mu} Q^{-1}\right)=\operatorname{rank}\left(\hat{F}_{\mu}\right)=\tilde{q}=\max \left\{q_{j}: j=1,2, \ldots, \sigma\right\} ;
$$

see also (1.17) and Proposition 2.1 of [9]. Moreover $\operatorname{rank}\left(\hat{F}_{\mu}^{\tilde{q}}\right)=p$.

Following Theorems 1.5 and 1.6, an analytic expression for the Drazin inverse of matrix $Q \hat{F}_{\mu} Q^{-1}$ is constructed:

$$
\left(Q \hat{F}_{\mu} Q^{-1}\right)^{D}=Q \hat{F}_{\mu}^{D} Q^{-1}=(I-X)[N \mid a]\left(Q \hat{F}_{\mu} Q^{-1}[a \mid b]\right)^{-1}(I-X)[b \mid N],
$$

where $Q \hat{F}_{\mu} Q^{-1}[a \mid b]$ is an $p \times p$ nonsingular submatrix of $Q \hat{F}_{\mu} Q^{-1}$ with row index set $a=\left\{i_{1}, i_{2}, \ldots, i_{p}\right\}$ and column index set $b=\left\{j_{1}, j_{2}, \ldots, j_{p}\right\}$. Obviously, the matrix $X=\left[\begin{array}{cc}\mathbb{O}_{p} & \mathbb{O}_{p, q} \\ \mathbb{O}_{q, p} & I_{p}\end{array}\right]$ satisfies condition (1.19), and $I-X=\left[\begin{array}{cc}I_{p} & \mathbb{O}_{p, q} \\ \mathbb{O}_{q, p} & \mathbb{O}_{q}\end{array}\right]$. Thus,

$$
(I-X)[N \mid a]=\left[\begin{array}{c}
I_{p} \\
\mathbb{O}_{q, p}
\end{array}\right],\left(I_{X}\right)[b \mid N]=\left[I_{p} \mathbb{O}_{p, q}\right]
$$

and

$$
\left(Q \hat{F}_{\mu} Q^{-1}[a \mid b]\right)^{-1}=\left(\mu I_{p}+J_{p}\right)
$$

Consequently,

$$
Q \hat{F}_{\mu}^{D} Q^{-1}=\left[\begin{array}{c}
I_{p} \\
\mathbb{O}_{q, p}
\end{array}\right]\left(\mu I_{p}+J_{p}\right)\left[I_{p} \mathbb{O}_{p, q}\right] \Rightarrow \hat{F}_{\mu}^{D}=Q\left[\begin{array}{cc}
\mu I_{p} & O_{p, q} \\
O_{q, p} & O_{q}
\end{array}\right] Q^{-1} .
$$


The matrix (3.2) is a Drazin inverse of $\hat{F}_{\mu}$.

According to the preceding discussion, the following theorem on the relation between the Drazin inverse and the Weierstrass canonical form of $F$ follows.

Proposition 3.4. The Drazin inverse of $F$ is given by

$$
F^{D}=Q\left[\begin{array}{rr}
J_{p} & O_{p, q} \\
O_{q, p} & O_{q}
\end{array}\right] Q^{-1}
$$

Proof. In practice, the Drazin inverse of $F$ is related to the study of the systems

$$
F \underline{x}^{\prime}(t)=I \underline{x}(t) \text {, or } F \underline{x}_{k+1}=I \underline{x}_{k},
$$

where $\operatorname{det} F=0$. Using Proposition 3.3 and letting $\mu=0$ and $G=1$, expression (3.3) is a straightforward result.

As a consequence, the following algorithmic approach follows.

Algorithm. Let $F, G \in \mathbb{C}^{n \times n}$.

1. Determine the Weierstrass canonical form of the matrix pencil $s F-G$.

Thus, index $[(\mu F+G) F]=q, \operatorname{rank}[(\mu F+G) F]=q, \operatorname{rank}[(\mu F+G) F]^{q}=p$, the index sets $a$ and $b, J_{p}$ and the nonsingular matrix $Q$ (and $Q^{-1}$ ) are all obtained.

2. Compute $I-X=\left[\begin{array}{cc}I_{p} & \mathbb{O}_{p, q} \\ \mathbb{O}_{q, p} & \mathbb{O}_{q}\end{array}\right]$ and submatrices $(I-X)[N \mid a]=\left[\begin{array}{c}I_{p} \\ \mathbb{O}_{q, p}\end{array}\right]$ and $(I-X)[b \mid N]=\left[I_{p} \mathbb{O}_{q, p}\right]$.

3. Finally, compute the Drazin inverse of $\hat{F}_{\mu}^{D}\left(\hat{F}_{\mu}\right)$

$$
\hat{F}_{\mu}^{D}=Q\left[\begin{array}{c}
I_{p} \\
\mathbb{O}_{q, p}
\end{array}\right]\left(\mu I_{p}+J_{p}\right)\left[I_{p} \mathbb{O}_{p, q}\right] Q^{-1}
$$

(In the special case that $\mu=0$ and $G=I$, we compute the Drazin inverse of $F$.)

EXAmPle 3.5. Let us determine the Drazin inverse of

$$
F=\left[\begin{array}{rrr}
2 & 0 & 0 \\
-1 & 1 & 1 \\
-1 & -1 & -1
\end{array}\right]
$$

For this purpose, consider the system $F \underline{x}^{\prime}(t)=\underline{x}(t)$ (or equivalently $F \underline{x}_{k+1}=I \underline{x}_{k}$ ), where $\operatorname{det} F=0$, and consider the matrix pencil given by

$$
s F-I=\left[\begin{array}{ccc}
2 s-1 & 0 & 0 \\
-s & s-1 & s \\
-s & -s & -s-1
\end{array}\right] .
$$


According to the detailed steps in the algorithm, firstly, we determine the Weierstrass canonical form for the matrix pencil above. We obtain the nonsingular $3 \times 3$ matrix $Q=\left[\begin{array}{rrr}1 & 0 & 0 \\ -1 & 1 & 1 \\ 0 & -1 & 0\end{array}\right], Q^{-1}=\left[\begin{array}{rrr}1 & 0 & 0 \\ 0 & 0 & -1 \\ 1 & 1 & 1\end{array}\right]$ and $J_{1}\left(\frac{1}{2}\right)=\frac{1}{2}$. Since index $(F)=2$, $\operatorname{rank}(F)=2$ and $\operatorname{rank}\left(F^{2}\right)=1$, we determine the index sets $a=\left\{i_{1}, i_{2}\right\}, b=\left\{j_{1}, j_{2}\right\}$.

According to the second step, we have

$$
(I-X)[N \mid a]=\left[\begin{array}{l}
1 \\
0 \\
0
\end{array}\right] \text { and }(I-X)[b \mid N]=\left[\begin{array}{lll}
1 & 0 & 0
\end{array}\right]
$$

and finally,

$$
\begin{aligned}
F^{D} & =Q\left[\begin{array}{c}
I_{1} \\
\mathbb{O}_{2,1}
\end{array}\right] J_{1}\left(\frac{1}{2}\right)\left[\begin{array}{ll}
I_{1} & \mathbb{O}_{1,2}
\end{array}\right] Q^{-1} \\
& =\left[\begin{array}{rrr}
1 & 0 & 0 \\
-1 & 1 & 1 \\
0 & -1 & 0
\end{array}\right]\left[\begin{array}{l}
1 \\
0 \\
0
\end{array}\right] \frac{1}{2}\left[\begin{array}{ll}
1 & 0
\end{array}\right]\left[\begin{array}{rrr}
1 & 0 & 0 \\
0 & 0 & -1 \\
1 & 1 & 1
\end{array}\right]=\left[\begin{array}{r}
1 \\
-1 \\
0
\end{array}\right] \frac{1}{2}\left[\begin{array}{lll}
1 & 0 & 0
\end{array}\right] \\
& =\frac{1}{2}\left[\begin{array}{rrr}
1 & 0 & 0 \\
-1 & 0 & 0 \\
0 & 0 & 0
\end{array}\right]
\end{aligned}
$$

Compare the result above with [2, pp. 126-127], [9, p. 204] and [11, pp. 187-188].

In the last part of this section, we present important core-nilpotent decomposition method.

Theorem 3.6 (Core-Nilpotent Decomposition Method). [2] For $F \in \mathbb{C}^{n \times n}$ with $\operatorname{Ind}(F)=k>0, \operatorname{dim} \Re\left(F^{k}\right)=s, \operatorname{dim} \aleph\left(F^{k}\right)=t(s+t=n)$, there exists a nonsingular matrix $T$ such that

$$
F=T\left[\begin{array}{cc}
C_{s} & \mathbb{O}_{s, t} \\
\mathbb{O}_{t, s} & N_{t}
\end{array}\right] T^{-1}
$$

where $C_{p}$ is a $p \times p$ nonsingular matrix and where $N_{q}$ is a $q \times q$ nilpotent matrix with index $k$.

Theorem 3.7. Let $\hat{F}_{\mu} \in \mathbb{C}^{n \times n}$ with $\operatorname{Ind}\left(\hat{F}_{\mu}\right)=k>0, \operatorname{dim} \Re\left(\hat{F}_{\mu}^{k}\right)=p, \operatorname{dim} \aleph\left(\hat{F}_{\mu}^{k}\right)$ $=q(p+q=n)$, and core-nilpotent decomposition

$$
\hat{F}_{\mu}=T_{\mu}\left[\begin{array}{cc}
C_{p, \mu} & \mathbb{O}_{p, q} \\
\mathbb{O}_{p, q} & N_{q, \mu}
\end{array}\right] T_{\mu}^{-1}
$$


for nonsingular matrices $T_{\mu}, C_{p, \mu}$ and a nilpotent matrix $N_{q, \mu}$ with index $K$, all of whom depend on the choice of $\mu \in \mathbb{C}$. Then there exist nonsingular matrices

$$
P_{\mu}=\left[\begin{array}{cc}
C_{p, \mu}^{-1} & \mathbb{O}_{p, q} \\
\mathbb{O}_{p, q} & \left(I-\mu N_{q, \mu}\right)^{-1}
\end{array}\right] T_{\mu}^{-1}(\mu F+G)^{-1}
$$

and

$$
Q_{\mu}=T_{\mu}
$$

such that the Weierstrass canonical form derives if and only if

$$
N_{q, \mu}=\left(I_{q}+\mu H_{q}\right)^{-1} H_{q}
$$

and

$$
C_{p, \mu}=\left(\mu I_{p}+J_{p}\right)^{-1}
$$

Proof. In order to construct the Weierstrass canonical form for the regular pencils, we should calculate

$$
\begin{aligned}
F_{w}=P_{\mu} F Q_{\mu} & =\left[\begin{array}{cc}
C_{p, \mu}^{-1} & \mathbb{O}_{p, q} \\
\mathbb{O}_{p, q} & \left(I_{q}-\mu N_{q, \mu}\right)^{-1}
\end{array}\right] T_{\mu}^{-1}(\mu F+G)^{-1} F T_{\mu} \\
& =\left[\begin{array}{cc}
C_{p, \mu}^{-1} & \mathbb{O}_{p, q} \\
\mathbb{O}_{p, q} & \left(I_{q}-\mu N_{q, \mu}\right)^{-1}
\end{array}\right] T_{\mu}^{-1} \hat{F}_{\mu} T_{\mu} \\
& =\left[\begin{array}{cc}
C_{p, \mu}^{-1} & \mathbb{O}_{p, q} \\
\mathbb{O}_{p, q} & \left(I_{q}-\mu N_{q, \mu}\right)^{-1}
\end{array}\right]\left[\begin{array}{cc}
C_{p, \mu} & \mathbb{O}_{p, q} \\
\mathbb{O}_{p, q} & N_{q, \mu}
\end{array}\right] \\
& =\left[\begin{array}{cc}
I_{p} & \mathbb{O}_{p, q} \\
\mathbb{O}_{p, q} & \left(I_{q}-\mu N_{q, \mu}\right)^{-1} N_{q, \mu}
\end{array}\right]
\end{aligned}
$$

and

$$
\begin{aligned}
G_{w}=P_{\mu} G Q_{\mu} & =\left[\begin{array}{cc}
C_{p, \mu}^{-1} & \mathbb{O}_{p, q} \\
\mathbb{O}_{p, q} & \left(I-\mu N_{q, \mu}\right)^{-1}
\end{array}\right] T_{\mu}^{-1}(\mu F+G)^{-1} G T_{\mu} \\
& =\left[\begin{array}{cc}
C_{p, \mu}^{-1} & \mathbb{O}_{p, q} \\
\mathbb{O}_{p, q} & \left(I-\mu N_{q, \mu}\right)^{-1}
\end{array}\right] T_{\mu}^{-1} \hat{G}_{\mu} T_{\mu} \\
& =\left[\begin{array}{cc}
C_{p, \mu}^{-1} & \mathbb{O}_{p, q} \\
\mathbb{O}_{p, q} & \left(I-\mu N_{q, \mu}\right)^{-1}
\end{array}\right]\left[\begin{array}{cc}
I_{p}-\mu C_{p, \mu} & \mathbb{O}_{p, q} \\
\mathbb{O}_{p, q} & I_{q}-\mu N_{q, \mu}
\end{array}\right] \\
& =\left[\begin{array}{cc}
C_{p, \mu}^{-1}\left(I_{p}-\mu C_{p, \mu}\right. & \mathbb{O}_{p, q} \\
\mathbb{O}_{p, q} & I_{q}
\end{array}\right] .
\end{aligned}
$$


Now, if we want to obtain the Weierstrass canonical form, then

$$
\begin{aligned}
\left(I_{q}-\mu N_{q, \mu}\right)^{-1} N_{q, \mu}=H_{q} & \Leftrightarrow N_{q, \mu}=\left(I_{q}-\mu N_{q, \mu}\right) H_{q} \\
& \Leftrightarrow N_{q, \mu}=H_{q}\left(I_{q}+\mu H_{q, \mu}\right)^{-1}=\left(I_{q}+\mu H_{q, \mu}\right)^{-1} H_{q}
\end{aligned}
$$

since we have already shown that the matrices $\left(I_{q}+\mu H_{q, \mu}\right)^{-1}, H_{q}$ commute. Moreover,

$$
\begin{aligned}
C_{p, \mu}^{-1}\left(I_{p}-\mu C_{p, \mu}\right)=J_{p} & \Leftrightarrow I_{p}-\mu C_{p, \mu}=C_{p, \mu} J_{p} \\
& \Leftrightarrow C_{p, \mu}\left(\mu I_{p}+J_{p}\right)=I_{p} \Leftrightarrow C_{p, \mu}=\left(\mu I_{p}+J_{p}\right)^{-1} .
\end{aligned}
$$

ExAMPLE 3.8. Consider the square system (1.1) (analogously with system (1.2))

$$
F \underline{x}^{\prime}(t)=G \underline{x}(t)
$$

with

$$
F=\left[\begin{array}{rrrr}
0 & 1 & -1 & 1 \\
0 & -2 & 2 & -1 \\
1 & 0 & 0 & 0 \\
0 & 0 & 0 & 0
\end{array}\right] \quad \text { and } \quad G=\left[\begin{array}{rrrr}
1 & 0 & 0 & 1 \\
1 & -1 & 1 & -1 \\
0 & 1 & 0 & 0 \\
1 & 0 & 0 & 0
\end{array}\right]
$$

If we set $\mu=1$, then $F+G=\left[\begin{array}{rrrr}-1 & 1 & -1 & 2 \\ 1 & -3 & 3 & -2 \\ 1 & 1 & 0 & 0 \\ 1 & 0 & 0 & 0\end{array}\right]$ is invertible and

$$
\hat{F}_{1}=(F+G)^{-1} F=\left[\begin{array}{rrrr}
0 & 0 & 0 & 0 \\
1 & 0 & 0 & 0 \\
1 & -\frac{1}{2} & \frac{1}{2} & 0 \\
0 & \frac{1}{4} & -\frac{1}{4} & \frac{1}{2}
\end{array}\right]
$$

Using the core-nilpotent decomposition method, we obtain the expression

$$
\hat{F}_{1}=\left[\begin{array}{rrrr}
0 & 0 & 0 & 0 \\
1 & 0 & 0 & 0 \\
1 & -\frac{1}{2} & \frac{1}{2} & 0 \\
0 & \frac{1}{4} & -\frac{1}{4} & \frac{1}{2}
\end{array}\right]=T=\left[\begin{array}{rrrr}
\frac{1}{2} & -\frac{1}{4} & 0 & 0 \\
0 & \frac{1}{2} & 0 & 0 \\
0 & 0 & 0 & 1 \\
0 & 0 & 0 & 0
\end{array}\right] T^{-1}
$$

where $T_{1}=\left[\begin{array}{llll}0 & 0 & 0 & 1 \\ 0 & 0 & 1 & 0 \\ 0 & 1 & 1 & 0 \\ 1 & 1 & 0 & 0\end{array}\right], T_{1}^{-1}=\left[\begin{array}{rrrr}0 & 1 & -1 & 1 \\ 0 & -1 & 1 & 0 \\ 0 & 1 & 0 & 0 \\ 1 & 0 & 0 & 0\end{array}\right], C_{2,1}=\left[\begin{array}{rr}\frac{1}{2} & -\frac{1}{4} \\ 0 & \frac{1}{2}\end{array}\right]$ and $N_{2,1}=\left[\begin{array}{ll}0 & 1 \\ 0 & 0\end{array}\right]$. 
Now, by using equations (3.6) and (3.7), we have

$$
\begin{aligned}
P_{1} & =\left[\begin{array}{ll}
{\left[\begin{array}{ll}
2 & 1 \\
0 & 2
\end{array}\right]} & \mathbb{O}_{2,2} \\
& \mathbb{O}_{2,2} \\
& {\left[\begin{array}{ll}
1 & 1 \\
0 & 1
\end{array}\right]}
\end{array}\right]\left[\begin{array}{rrrr}
0 & 1 & -1 & 1 \\
0 & -1 & 1 & 0 \\
0 & 1 & 0 & 0 \\
1 & 0 & 0 & 0
\end{array}\right]\left[\begin{array}{rrrr}
-1 & -3 & 3 & -2 \\
3 & 7 & -7 & 4 \\
0 & -1 & 1 & 0 \\
0 & 1 & -1 & 1
\end{array}\right] \\
& =\left[\begin{array}{llll}
1 & 0 & 0 & 1 \\
1 & 1 & 0 & 0 \\
0 & 0 & 1 & 0 \\
0 & 0 & 0 & 1
\end{array}\right]
\end{aligned}
$$

and

$$
Q_{1}=T_{1}=\left[\begin{array}{cccc}
0 & 0 & 0 & 1 \\
0 & 0 & 1 & 0 \\
0 & 1 & 1 & 0 \\
1 & 1 & 0 & 0
\end{array}\right]
$$

Finally, the Weierstrass canonical form derives. Specifically,

$$
\begin{aligned}
P_{1} F Q_{1} & =\left[\begin{array}{llll}
1 & 0 & 0 & 1 \\
1 & 1 & 0 & 0 \\
0 & 0 & 1 & 0 \\
0 & 0 & 0 & 1
\end{array}\right]\left[\begin{array}{rrrr}
0 & 1 & -1 & 1 \\
0 & -2 & 2 & -1 \\
1 & 0 & 0 & 0 \\
0 & 0 & 0 & 0
\end{array}\right]\left[\begin{array}{llll}
0 & 0 & 0 & 1 \\
0 & 0 & 1 & 0 \\
0 & 1 & 1 & 0 \\
1 & 1 & 0 & 0
\end{array}\right] \\
& =\left[\begin{array}{llll}
1 & 0 & 0 & 0 \\
0 & 1 & 0 & 0 \\
0 & 0 & 0 & 1 \\
0 & 0 & 0 & 0
\end{array}\right]=F_{w}
\end{aligned}
$$

and

$$
\begin{aligned}
P_{1} G Q_{1} & =\left[\begin{array}{llll}
1 & 0 & 0 & 1 \\
1 & 1 & 0 & 0 \\
0 & 0 & 1 & 0 \\
0 & 0 & 0 & 1
\end{array}\right]\left[\begin{array}{rrrr}
-1 & 0 & 0 & 1 \\
1 & -1 & 1 & -1 \\
0 & 1 & 0 & 0 \\
1 & 0 & 0 & 0
\end{array}\right]\left[\begin{array}{llll}
0 & 0 & 0 & 1 \\
0 & 0 & 1 & 0 \\
0 & 1 & 1 & 0 \\
1 & 1 & 0 & 0
\end{array}\right] \\
& =\left[\begin{array}{llll}
1 & 1 & 0 & 0 \\
0 & 1 & 0 & 0 \\
0 & 0 & 1 & 0 \\
0 & 0 & 0 & 1
\end{array}\right]=G_{w} .
\end{aligned}
$$

Moreover, we can easily verify expressions (3.8) and (3.9).

$$
\begin{aligned}
& N_{2,1}=\left(I_{2}+H_{2}\right)^{-1} H_{2}=\left[\begin{array}{ll}
0 & 1 \\
0 & 0
\end{array}\right] \quad \text { and } \quad H_{2}=\left(I_{2}-N_{2,1}\right)^{-1} N_{2,1}=\left[\begin{array}{ll}
0 & 1 \\
0 & 0
\end{array}\right] \\
& C_{2,1}=\left(I_{2}+J_{2}(1)\right)^{-1}=\left[\begin{array}{ll}
2 & 1 \\
0 & 2
\end{array}\right] \quad \text { and } \quad J_{2}(1)=C_{2,1}^{-1}\left(I_{2}-C_{2,1}\right)=\left[\begin{array}{ll}
1 & 1 \\
0 & 1
\end{array}\right] .
\end{aligned}
$$


Acknowledgments. The authors are very grateful to Associate Managing Editor M. Tsatsomeros and an anonymous referee for their insightful comments, which improved the quality of the paper.

\section{REFERENCES}

[1] A. Ben-Israel and T.N.E. Greville. Generalized Inverses: Theory and Applications. John Wiley and Sons, Inc, 1974.

[2] S.L. Campbell. Singular Systems of Differential Equations. Pitman (Advanced Publishing Program), Great Britain, Vol. 1 (1980), Vol. 2 (1982).

[3] S.L. Campbell and C.D. Meyer. Generalized Inverses of Linear Transformations. Dover Publications, New York, 1991.

[4] D.G. Forney. Minimal bases of rational vector spaces with application to multivariable systems. SIAM Journal of Control, 13:505-514, 1975.

[5] M. Friedler and T.L. Markham. A characterization of the Moore-Penrose inverse. Linear Algebra and its Applications, 179:129-133, 1993.

[6] R.F. Gantmacher. The Theory of Matrices, Vol. I and II. Chelsea, New York, 1959.

[7] E. Grispos, Singular generalized autonomous linear differential systems. Bulletin of Greek Mathematical Society, 34:25-43, 1992.

[8] G.I. Kalogeropoulos. Matrix Pencils and Linear Systems. Ph.D Thesis, City University, London, 1985.

[9] G.I. Kalogeropoulos, A.D. Karageorgos, and A.A. Pantelous. On the relation of the Drazin inverse and matrix pencil theory for the study of generalized linear systems. Linear Algebra and its Applications, 427:197-205, 2007.

[10] V. Lovass-Nagy and D.L. Powers. On rectangular systems of differential equations and their application to circuit theory. Journal of Franklin Institute, 299(6):399-407, 1975.

[11] L. Zhang. A characterization of the Drazin inverse. Linear Algebra and its Applications, 335:183-188, 2001. 